\title{
Lactoferrin-Conjugated Polylactic Acid Nanobubbles Encapsulated Perfluoropentane as a Contrast Agent for Ultrasound/Magnetic Resonance Dual-Modality Imaging
}

\author{
Liqiong Ding ${ }^{1, \#}$, Pingsheng $\mathrm{Li}^{2, \#}$, Liu $\mathrm{He}^{1}$, Fengnan $\mathrm{Xu}^{1}$, Jieqiong Ding ${ }^{3, *}$ and Binhua $\mathrm{Luo}^{1, *}$ \\ ${ }^{1}$ School of Pharmacy, Hubei University of Science and Technology, Xianning, 437100, China \\ ${ }^{2}$ Pharmacy Department of the Second Affiliated Hospital, Hubei University of Science and Technology, Xianning, 437100, China \\ ${ }^{3}$ School of Basic Medical Sciences, Hubei University of Science and Technology, Xianning, 437100, China \\ *Corresponding Authors: Jieqiong Ding. Email: djq197911@163.com; Binhua Luo. Email: lbh9811015@163.com \\ "Equal contributors
}

Received: 07 April 2021 Accepted: 29 June 2021

\begin{abstract}
The development of contrast agents that can be activated by multiple modes is of great significance for tumor diagnosis. In this study, the lactoferrin (Lf)-conjugated polylactic acid (PLLA) nanobubbles (Lf-PLLA NBs) were used to encapsulate liquid perfluoropentane (PFP) with the double emulsion method, creating PFP loaded (PFP/ Lf-PLLA) NBs for the ultrasound/magnetic resonance dual-modality imaging of subcutaneous tumor. The particle diameter and stability of nanobubbles were investigated by photon correlation spectroscopy. The biocompatibility of nanobubbles was preliminarily evaluated by cell proliferation and migration assay, hemolysis rate, and blood biochemistry analysis. A B-mode clinical ultrasound real-time imaging system was used to perform ultrasonic imaging in vivo. Magnetic resonance imaging in vivo was applied with a clinical $3.0 \mathrm{~T}$ magnetic resonance imaging (MRI) scanner system. The mean particle diameter of PFP/Lf-PLLA NBs was $320.2 \pm 4.1 \mathrm{~nm}$ with a low polydispersity index (PDI, $0.145 \pm 0.025)$, and the NBs were negatively charged $(-11.4 \pm 0.4 \mathrm{mV})$. The transmission electron microscopy (TEM) results showed that PFP/Lf-PLLA NBs exhibited highly monodispersed and possessed an obvious spherical structure of nanocapsules. Nanobubbles had good stability at $4^{\circ} \mathrm{C}$. Different concentrations of the PFP/Lf-PLLA NBs solution had no effect on the cell in cytotoxicity and cell migration, and the results of hemolysis rate and blood biochemistry assay also indicated the good biocompatibility of NBs. On the ultrasound/magnetic resonance imaging of tumor-bearing mice, PFP/Lf-PLLA NBs showed significantly enhanced contrast ability of tumor tissue. Therefore, PFP/Lf-PLLA NBs had great potential to be a contrast agent for tumor dual-modality imaging in vivo.
\end{abstract}

\section{KEYWORDS}

Lactoferrin; polylactic acid; nanobubbles; contrast agent; ultrasound/magnetic resonance imaging

\section{Introduction}

Medical imaging techniques, such as magnetic resonance imaging (MRI), ultrasound imaging and fluorescence imaging technology, were used to diagnose the tumor, which can represent an integrated tissue property with a certain dimension or a cross-sectional slice and depict the inner structure [1]. 
However, to a certain extent, each technology presents its advantages and disadvantages. In recent years, multimodal imaging plays a particularly important role in the early diagnosis, treatment monitoring and prognosis prediction of diseases. Ultrasound and magnetic resonance imaging are both non-ionizing in nature and considered hazardless measurements in standard clinical practice [2-4]. They can measure the blood flow velocity and blood flow dynamics evaluating the function of tissue or organ. Furthermore, they all need contrast agents, which are frequently used to enhance the contrast between normal and abnormal tissue for correct and early detection of pathology.

Microbubbles have been used to enhance acoustic backscattering signals in ultrasound imaging for more than 20 years, which are used as a contrast agent for tumor ultrasound imaging, or a drug carrier that is delivered to the targeted tissue [5-7]. The reflection intensity of ultrasonic is related to the acoustic impedance of the two media at the phase interface, and the acoustic impedance of the microbubbles is much higher than that of tissue or fluid inside the body. This difference serves as a theoretical underpinning for organ edge delineation and perfusion imaging and obtains a higher image resolution. Furthermore, the potential application of microbubbles used as a unique magnetic resonance susceptibility contrast agent was based on the induction of large local magnetic susceptibility differences by the gasliquid interface [8-12]. In recent years, potential applications of microbubbles as magnetic resonance pressure sensors have been investigated based on pressure-induced sensitivity changes. The $T_{2^{-}}$and $T^{*}{ }_{2^{-}}$ based measurements of pressure are strongly influenced by the bubble size at atmospheric pressure, static magnetic field strength, the magnitude of the susceptibility difference between the encapsulated gas and plasma, bubble volume fraction, and the refocusing interval [10].

However, the diameters of microbubbles are much larger (about $2 \sim 8 \mu \mathrm{m}$ ), which cannot pass through tumor vasculature endothelial gaps and only stay in the circulation to behave as blood-pool markers [13]. In order to overcome this problem, nanobubbles have been developed due to their potential for US imaging outside blood vessels, which has caught the researcher's attention [14-16]. The vascular endothelial gap in tumors is approximately $380 \sim 760 \mathrm{~nm}$, which is much wider than that of normal tissue (the endothelial gap is less than $7 \mathrm{~nm}$ ) [17]. Nanobubbles (NBs) are nanosized bubbles and can effectively accumulate in a tumor due to the enhanced permeability and retention (EPR) effects, which are promising contrast agents for their ability to penetrate tumor blood vessel pores to allow for targeted imaging [18-21].

In recent years, nanobubbles consisted of surfactants, lipids, albumin, and biocompatible polymers have been used as a contrast agent in ultrasound imaging and exhibited good contrast enhancement [22-26]. The most important biodegradable polymers, such as polylactic acid (PLA) and polyglycolic acid (PGA), have wide application prospects in the field of biomedicine [27-30]. In addition, degraded monomers are easy to be metabolized through the normal metabolic pathways and harmless to the human body [31].

In this study, perfluoropentane (PFP) loaded polylactic acid (PLLA) nanobubbles (PFP/PLLA NBs) were prepared by the double emulsion method. Lactoferrin (Lf) as a ligand for targeting the glioma cells was conjugated with nanobubbles (PFP/Lf-PLLA NBs). The appearance, average diameter, zeta potential and stability of the obtained PFP/Lf-PLLA NBs were studied. The biocompatibility of PFP/Lf-PLLA NBs was evaluated by cytotoxicity, cell migration and blood biochemical analysis. In order to carry out extravascular imaging, and overcome the shortcoming that micron contrast agent can only be used as blood pool contrast agent, PFP/Lf-PLLA NBs as a potentially promising multimodal nanoscale contrast agent for ultrasound/magnetic resonance imaging was performed on $\mathrm{BALB} / \mathrm{c}$ nude mice bearing subcutaneous tumors.

\section{Materials and Methods}

Poly(vinyl alcohol) was obtained from Aladdin Reagent Co., Ltd. (Shanghai, China) and used as an emulsifier. Perfluoropentane was obtained from Jenkem Technology Co, Ltd. (Beijing, China), L-lactide was purchased from Daigang Biomaterial Co., Ltd. (Jinan, China), which were used to prepare 
nanobubbles. Lactoferrin (Lf) was obtained from Sigma Chemical Co. (St Louis, MO, USA) and used as a targeted group. Rat C6 glioma (C6) cells and human normal liver cells (HL-7702) were obtained from the Institute of Life Science Cell Culture Center (Shanghai, China) and used for cell experiments and animal model construction.

\subsection{Preparation of Targeted PFP/Lf-PLLA NBs}

The PFP/Lf-PLLA NBs were developed with a traditional double-emulsion method. PLLA (50 mg) was dissolved in a plastic tube containing chloroform $(1 \mathrm{~mL})$ and the solution was used as the $\mathrm{O}_{2}$ phase. After PFP $(0.5 \mathrm{~mL})$ was added, the mixture was stirred for $3 \mathrm{~min}$ at $10,000 \mathrm{rpm}$ to get the primary $\mathrm{O}_{1} / \mathrm{O}_{2}$ emulsion. Afterward, the primary $\mathrm{O}_{1} / \mathrm{O}_{2}$ emulsion was added to a tube containing $0.5 \%$ PVA solution $(10 \mathrm{~mL})$ and sonicated at low temperature for $5 \mathrm{~min}$ to get $\mathrm{O}_{1} / \mathrm{O}_{2} / \mathrm{W}$ double emulsion. After continuous stirring for a period of $24 \mathrm{~h}$ at $25^{\circ} \mathrm{C}$, the organic solvent evaporated, and NBs were obtained. The PFP/PLLA NBs were centrifuged at high speed $(12000 \mathrm{rpm}, 5 \mathrm{~min})$ and the precipitate was redispersed in normal saline solution (NS). Then, NBs suspension was poured into $\alpha$-Malemidyl- $\omega-\mathrm{N}-$ Hydroxysuccinimidyl poly(ethyleneglycol) solution, and incubate with lactoferritin sulfhydryl group in PBS under gentle stirring. Therefore, lactoferrin as a ligand for targeting the glioma was conjugated with PFP/PLLA NBs. The method was performed in detail as described in our previously published works [32]. The flowchart of preparation was shown in Scheme 1.
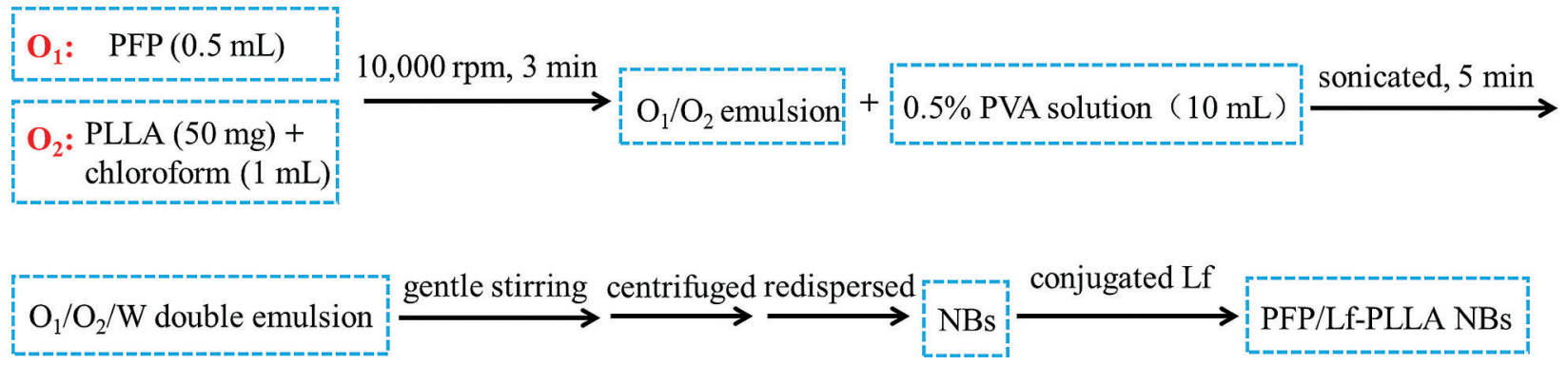

Scheme 1: Flowchart of the PFP/Lf-PLLA NBs preparation

\subsection{Characteristics of PFP/Lf-PLLA NBs}

The structure and morphology of the obtained PFP/Lf-PLLA NBs were characterized by Transmission Electron Microscope (TEM, JEM-2010, Japan). The diluted $\mathrm{NB}_{\mathrm{S}}$ was sprayed on the copper net, which was dried naturally and dyed with $2 \%$ phosphotungstic acid aqueous solution.

The particle diameter and zeta potential of the PFP/Lf-PLLA NBs were measured by laser particle size analyzer (Zetasizer Nano ZSE, Malvern). The PFP/Lf-PLLA NBs were diluted with distilled water and measured three times at $25^{\circ} \mathrm{C}$. The obtained results were average values.

The PFP/Lf-PLLA NBs solution was stored at $4^{\circ} \mathrm{C}$. Within a specified period $(0,5,10,15,20$ days), the samples of PFP/Lf-PLLA NBs solution were taken out of the refrigerator, and after rising to room temperature, the samples were diluted with deionized water to obtain a suitable concentration. Then, the average hydrodynamic diameter, zeta potential and particle diameter distribution of the obtained samples were measured with a laser particle size analyzer at $25^{\circ} \mathrm{C}$, and each experiment was repeated three times under the same conditions.

\subsection{Cytotoxicity Assay}

HL-7702 cells were used for cell viability studies determined by the MTT assay. After resuscitation, the cells were cultured in Dulbecco's modified Eagle medium (DMEM) supplemented with 10\% fetal bovine 
serum. After passaged 3 times, cells were seeded on a 96-well plate at a density of 5000 cells to each well, and cultured at $37^{\circ} \mathrm{C}$ in a humidified $5 \% \mathrm{CO}_{2}$ cell incubator for $24 \mathrm{~h}$. Afterwards, the PFP/Lf-PLLA NBs were poured into the cell medium at concentrations of $0.5 \%, 1.0 \%, 2.0 \%, 3.0 \%, 4.0 \%$, and $5.0 \%(\mathrm{v} / \mathrm{v})$ and cells incubated with medium for another $24 \mathrm{~h}$. PBS was used as the control group. Then, $20 \mu \mathrm{L}$ of the prepared MTT solution $(5.0 \mathrm{mg} / \mathrm{mL})$ was added, and the cells were incubated for another $4 \mathrm{~h}$. Subsequently, the medium was removed and the formazan crystals were dissolved by dimethyl sulfoxide. The absorbance value was measured by a microplate reader (Bio-Tek Instruments, Winooski, VT, USA) at $570 \mathrm{~nm}$, and calculated the cell survival rate according to the following formula:

Cell viability $=\frac{A_{\text {sample }}-A_{\text {blank }}}{A_{\text {contrast }}-A_{\text {blank }}} \times 100 \%$

where the $A_{\text {sample }}$ is the cells solution containing nanobubbles, the $A_{\text {blank }}$ is the PBS, and the $A_{\text {contrast }}$ is the cells solution without nanobubbles.

\subsection{Determination of Hemolytic Activity}

After the Sprague Dawley rats (about $200 \mathrm{~g}, \mathrm{n}=3$ ) were anesthetized with 4\% chloral hydrate, the femoral artery was exposed, and a catheter was inserted. Blood samples were obtained from the femoral arteries and placed in test tubes bathed with heparin sodium. After centrifugation, the supernatant was discarded and the red blood cells were collected. The collected erythrocytes were washed three times and re-dispersed in normal saline $(2 \%, \mathrm{v} / \mathrm{v})$. The PFP/Lf-PLLA NB $\mathrm{N}_{\mathrm{S}}$ solution was mixed with the erythrocytes suspension and different concentrations samples $(0.5 \%, 1 \%, 2 \%, 3 \%, 4 \%$ and $5 \%)$ were formed. The negative control group was the solution of erythrocytes suspension $(150 \mu \mathrm{L})$ and normal saline $(150 \mu \mathrm{L})$, the positive control group was a mixture of double-distilled water $(150 \mu \mathrm{L})$ and erythrocytes suspension $(150 \mu \mathrm{L})$. The samples were incubated for $1 \mathrm{~h}$ at $37^{\circ} \mathrm{C}$ and then centrifuged at $3500 \mathrm{rpm}$ for $25 \mathrm{~min}$. The measurement of the optical density of the supernatants was carried out on a spectrophotometer at $540 \mathrm{~nm}$. The calculation formula of hemolysis rate is as follows.

Hemolysis ratio $=\frac{A_{\text {sample }}-A_{\text {negative }}}{A_{\text {positive }}-A_{\text {negative }}} \times 100 \%$

\subsection{Cell Migration Assay}

The scratch assay was carried out to analyze cell migration ability in vitro. C6 glioma cells were seeded in six-well plates at a density of $6 \times 10^{5}$ cells per well, and cultured overnight at $37^{\circ} \mathrm{C}$ in a $5 \% \mathrm{CO}_{2}$ humidified incubator. After the cells became adherent and the density was more than $90 \%$, which were scratched in a straight line with a $200 \mu \mathrm{L}$ pipette tip, and floating debris or dead cells were removed by gentle washing with PBS. The cells were cultured in the medium containing the PFP/Lf-PLLA NBs with different concentrations for $24 \mathrm{~h}$. The photographic images were taken using an inverted microscope (Olympus, Japan) at different time points. The images were analyzed by Image J software.

\subsection{Blood Biochemical Analysis}

Five adult male New Zealand white rabbits, weighing $2.5 \sim 3.0 \mathrm{~kg}$, were randomly selected for this study. The rabbits were injected intravenously through the marginal ear vein with $4 \%$ chloral hydrate. Blood samples $(2 \mathrm{~mL})$ were withdrawn from the heart of the anesthetized rabbits through a syringe and used as the control group. Then, animals were injected intravenously with PFP/Lf-PLLA NBs at a dose of $2 \mathrm{~mL} / \mathrm{kg}$. After $2 \mathrm{~h}$, blood samples were collected for biochemical analysis. 


\subsection{Use of the PFP/Lf-PLLA NBs for Ultrasound/Magnetic Resonance-Enhanced Tumor Imaging in Vivo}

All animal experiments were performed according to the guidelines issued by the Hubei University of Science and Technology, which are in accordance with the National Institutes of Health (NIH) guidelines.

Male BALB/c nude mice, aged 4 weeks and weighing 18-20 g, were purchased from the Animals Center of Tongji Medical University. The collected C6 glioma cells (about $2.5 \times 106$ cells) were resuspended in $100 \mu \mathrm{L}$ phosphate-buffered saline and inoculated subcutaneously into nude mice to develop a nude mouse model of subcutaneous glioma. The experiments of ultrasound/magnetic resonance-enhanced tumor imaging in vivo were then performed when the mean tumor size was $0.8 \mathrm{~cm}$. The subcutaneous tumor models of nude mice $(\mathrm{n}=6)$ were anesthetized via an intraperitoneal injection of $4 \%$ chloral hydrate. Firstly, the $10 \mathrm{MHz}$ probe (Philips IU22, Amsterdam, Netherlands) was coated with a layer of ultrasonic medicinal coupling gel, which would prevent the presence of air, and then placed on the surface of the tumor to acquire the ultrasound images, which were used as a control. Afterward, PFP/Lf-PLLA NBs $\left(100 \mu \mathrm{L}, 1.5 \times 10^{8}\right.$ bubbles $/ \mathrm{mL}$ ) were directly injected into the tumor site of the tumor-bearing mice and collected the images. All contrast images were recorded on B-mode imaging, with the persistence of low, the mechanical index (MI) was 0.06 , the frame rate was $37 \mathrm{~Hz}$, the dynamic range was $55 \mathrm{~dB}$, and the gain was $67 \%$. Magnetic resonance imaging experiments were performed with an animal coil on a 3.0-T clinical whole-body magnetic resonance imaging scanner (General Electric Medical Systems, USA). Before the administration, the mice were anesthetized. The mice were scanned before and after the administration of PFP/Lf-PLLA NBs solution, T2-weighted images were acquired using a spin-echo sequence. The scanning parameters were as follows: $\mathrm{TR} / \mathrm{TE}=4594 \mathrm{~ms} / 95.26 \mathrm{~ms}$, matrix $=256 \times 256$, field of view $(\mathrm{FOV})=60 \times 60 \mathrm{~mm}$, slice thickness $=1.4 \mathrm{~mm}$, Average: 6 (2DT2WI TSE sequence).

\subsection{Statistical Analysis}

All data were expressed as mean $\pm \mathrm{SD}$. The data analysis was performed using Student's $t$-tests.

\section{Results and Discussion}

\subsection{Preparation and Characterization of PFP/Lf-PLLA NBS}

Nanobubbles were manufactured by a double-emulsion (water/oil/water) and solvent-evaporation method described in our previous works [32]. The outer shell of the nanobubble was made of polylactic acid (PLLA), and the core of nanobubbles was liquid PFP, which was wrapped with the outer layer of PLLA, and a core-shell structure was developed. Liquid PFP with the boiling point of $28.5^{\circ} \mathrm{C}$ at atmospheric pressure was encapsulated by hydrophobic polylactic acid, which would retard the outward diffusion of PFP and enhanced storage stability, in addition, compared with other gases, the encapsulated liquid PFP would improve the storage stability of the nanobubbles. The PFP/Lf-PLLA NBs were obtained by conjugating lactoferrin to the surface of nanobubbles.

The characterization of PFP/Lf-PLLA NBs was shown in Fig. 1. The nanobubble solution was milky white at room temperature (Fig. 1a). The mean diameter of PFP/Lf-PLLA NBs was $320.2 \pm 4.1 \mathrm{~nm}$ (Fig. 1b) and the polydispersity index (PDI) was $0.145 \pm 0.025$, revealing the uniform size and good dispersion of PFP/Lf-PLLA NBs. Furthermore, the zeta potential of the NBs was $-11.4 \pm 0.4 \mathrm{mV}$. The TEM images of the nanobubbles were shown in Fig. 1c, which revealed that PFP/Lf-PLLA NBs had good dispersion and no aggregation. The nanobubbles were spherical in shape and had an obvious capsular structure $[33,34]$.

\subsection{Stability of PFP/Lf-PLLA NBs in Vitro}

The nanobubbles were nanoscale particle size with a high surface area to volume ratio, which resulted in high surface Gibbs free energy. They might be prone to aggregation and more instability during long periods of storage. Once the nanobubbles aggregated together during storage periods, their hydrodynamic diameter 
or the zeta potential should be changed. So the stability in vitro of nanobubbles could be estimated by measuring their changes of zeta potential and hydrodynamic diameter. The aqueous dispersion of PFP/LfPLLA NBs was placed at $4{ }^{\circ} \mathrm{C}$ in a refrigerator and measured with a laser particle size analyzer at $25^{\circ} \mathrm{C}$ during the experiment $(0,5,10,15,20$ days). As shown in Fig. 2, the changes of nanobubbles in particle diameter and zeta potential were very few, which illustrated that the PFP/Lf-PLLA NBs exhibited good stability in vitro. The PLLA was used as a shell, and PFP was encapsulated with PLLA. The core-shell structure improves the stability of the capsules, compared to that stabilized by a monomolecular layer of surfactant [35].

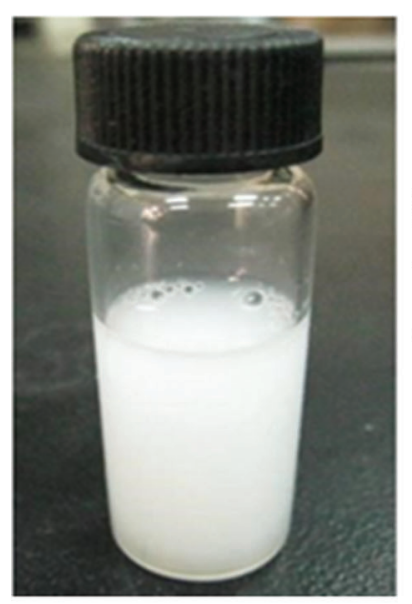

(a)

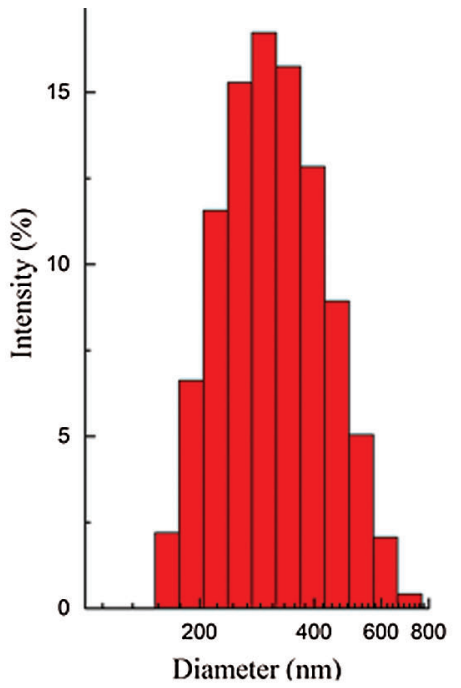

(b)

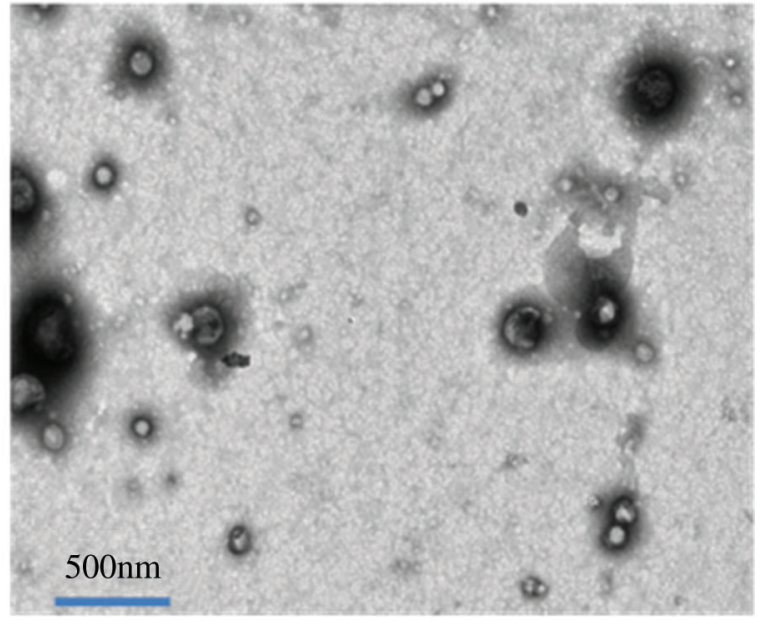

(c)

Figure 1: Characteristics of PFP/Lf-PLLA nanobubbles. (a) photograph (b) particle diameter distribution, and (c) transmission electron microscopy image of PFP/Lf-PLLA nanobubbles
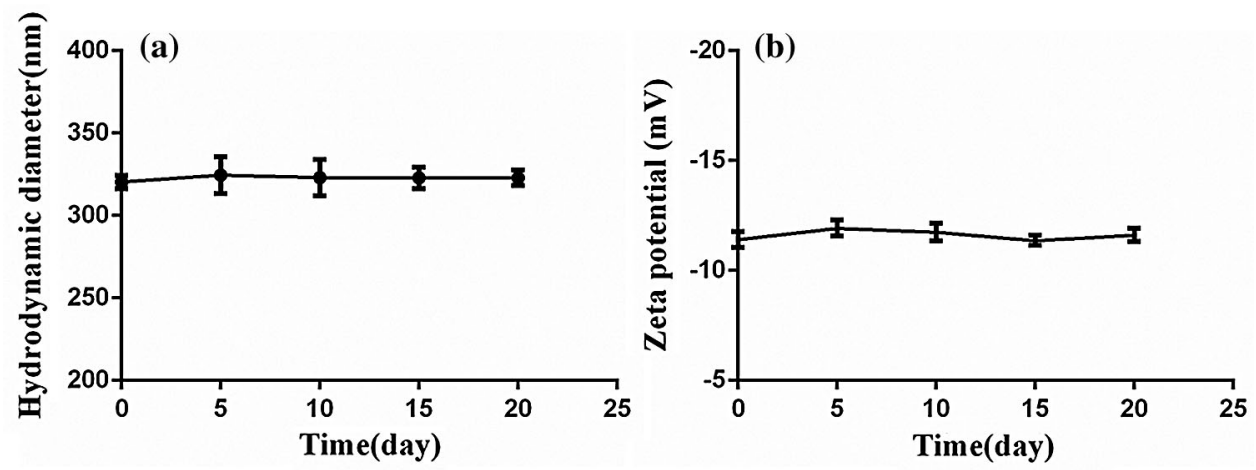

Figure 2: The stability of PFP/Lf-PLLA NBs in vitro. (a) The particle diameter and (b) zeta potential of PFP/ Lf-PLLA NBs

\subsection{Cytotoxicity Assay and Hemolysis of PFP/Lf-PLLA NBs}

Cytotoxicity is a method to evaluate the biocompatibility of nanomaterials. When the material enters the body, it will interact with normal cells. As a kind of normal cells, HL7702 is used to evaluate the interaction 
between nanobubbles and normal cells to see whether they inhibit the growth of normal cells, and to judge the cytotoxic effect of the material through this experiment. The cytotoxicity effect of PFP/Lf-PLLA NBs at $37^{\circ} \mathrm{C}$ for $24 \mathrm{~h}$ was determined by MTT assay. At the evaluated concentration, the cytotoxic effect of PFP/LfPLLA NBs to the HL7702 cells was less prominent and the cell viability was more than $90 \%$ (Fig. 3a). Furthermore, the hemolysis rates were less than 5\% within the evaluated concentration range (Fig. $3 \mathrm{~b}$ ), which suggested that the PFP/Lf-PLLA NBs had no hemolysis effect. The above experimental results indicated that the PFP/Lf-PLLA NBs had great potential in the biomedical field as a carrier.

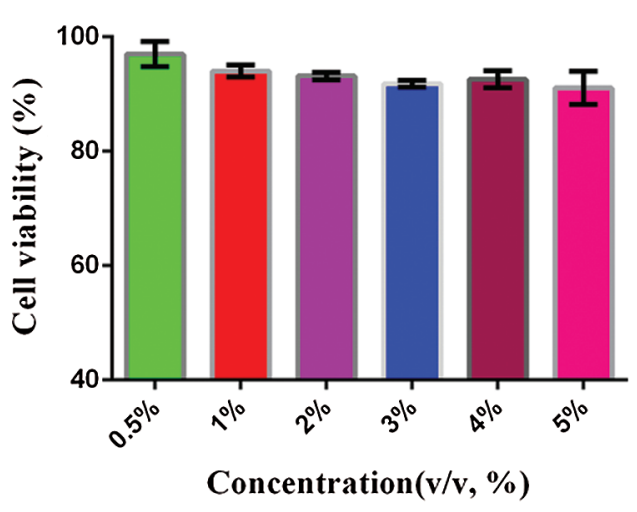

(a)

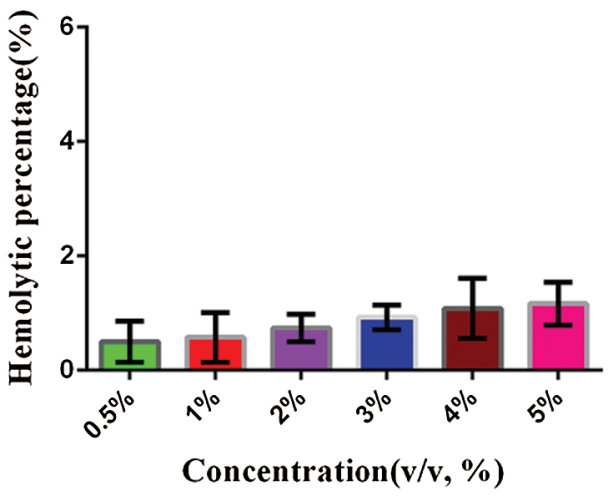

(b)

Figure 3: The cytotoxicity and hemolysis of PFP/Lf-PLLA NBs. (a) in vitro cytotoxicity assays, HL7702 cells were treated with PFP/Lf-PLLA NBs at different doses for $24 \mathrm{~h}$. (b) in vitro hemolysis, the hemolysis rate of PFP/LF-PLLA $\mathrm{NB}_{\mathrm{S}}$ interacting with erythrocyte suspension. Data were reported as mean $\pm \operatorname{SD}(n=5)$

\subsection{Cell Migration Assay}

Cell scratch assay is a straightforward and well-developed method, which is particularly suitable for researches on the effects of the extracellular matrix on cell migration. Cell scratch assay was used to detect the effect of PFP/LF-PLLA NBS as extracellular matrix on cell migration. Under the experimental concentration of PFP/Lf-PLLA NBs (1\%, 5\%), after the artificial gap was formed, the cells on the edge of the newly created gap began to move toward the opening to close the artificial gap, and after $24 \mathrm{~h}$ of incubation, the cell-cell contacts were established again (Fig. 4).

The cell migration rate was calculated as follows:

Migration rate $=\left[\left(\operatorname{Area}_{(0)}-\operatorname{Area}_{(\mathrm{t})}\right) / \operatorname{Area}_{(0)}\right] \times 100 \%$

The cell migration rate of the blank control group (without the PFP/Lf-PLLA NBs), the experimental groups $(1 \%, 5 \%$, v/v) were about $76.52 \pm 2.326 \%, 74.92 \pm 1.986 \%, 72.9 \pm 2.389 \%(\mathrm{n}=3)$, respectively. Compared with the blank control group, there was no significant change in the migration ability of C6 glioma cells in the experimental groups within $24 \mathrm{~h}$, the results were shown in Fig. 5. Statistical analysis showed that there was no significant difference among all groups. The results showed that PFP/Lf-PLLA $\mathrm{NB}_{\mathrm{S}}$ had no significant effect on the migration and growth of $\mathrm{C} 6$ glioma cells, which confirmed the good biocompatibility of PFP/LF-PLLA NB . $_{S}$ 


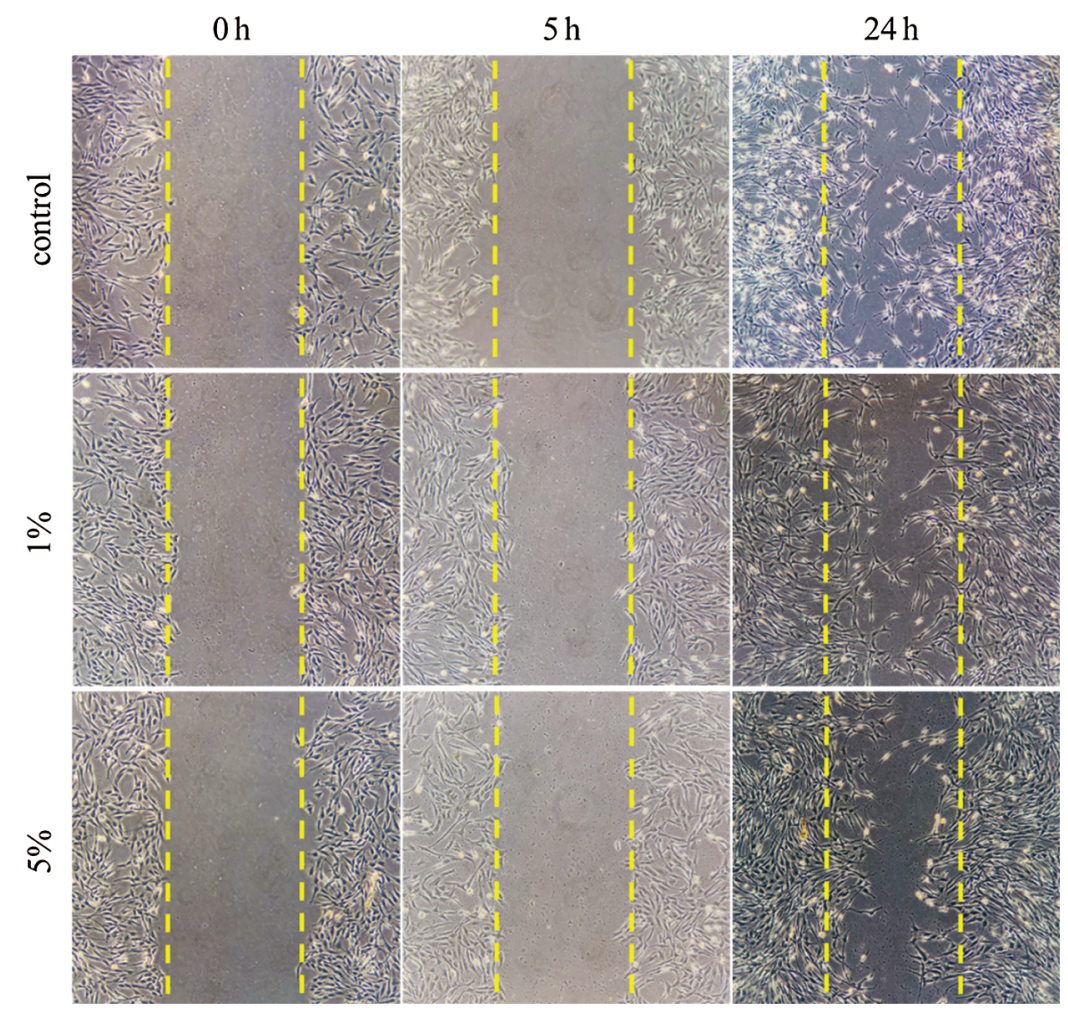

Figure 4: Effects of different concentrations of PFP/Lf-PLLA NBs on the migration of C6 glioma cells

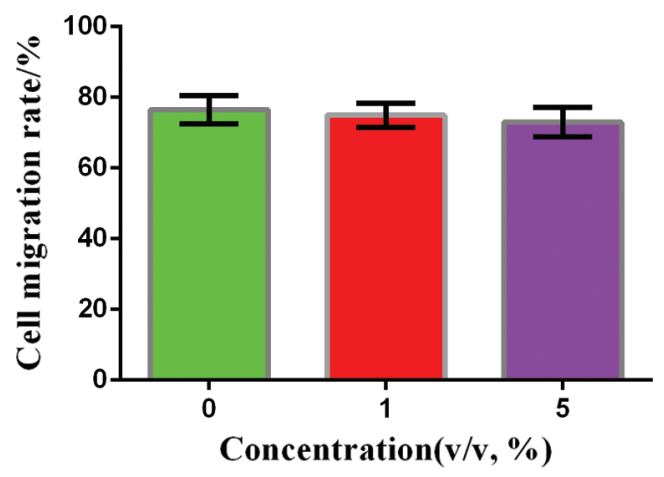

Figure 5: Migration rate of C6 glioma cells with different PFP/Lf-PLLA NBs concentrations after $24 \mathrm{~h}$ incubation

\subsection{Biochemical Analysis}

Hematology and blood biochemistry test are frequently used not only to assess overall health but also to monitor animals' effectiveness and safety of interventions including treatment, drugs, and other exogenous substances. If the toxic substance causes the body's tissues to fail to function properly, then the level of some enzymes in that tissue will change dramatically. The rabbits were anesthetized and the total blood was collected via cardiac puncture before and $2 \mathrm{~h}$ after the injection of nanobubbles and performed for hematology and biochemistry test. The serum was used to examine uric acid and creatinine as two renal function indicators, aspirate aminotransferase (AST), alanine transaminase (ALT), alkaline phosphatase (AP), the total protein, total bilirubin, albumin propagated, direct bilirubin as seven important liver 
function indicators. Fig. 6a illustrated the liver function, no significant differences were detected in AST, ALT and AP values before and after injection of nanobubbles, and the values of the total protein, total bilirubin, albumin propagated and direct bilirubin changed little during the experiment $(P>0.05)$. Similarly, as shown in Fig. 6b, no significant differences were detected in uric acid and creatinine values before and after injection of nanobubbles $(P>0.05)$. The above results indicated that nanobubbles had no obvious potential toxicity to the liver and kidneys. However, gadolinium-based contrast agents have been reported to be associated with an increased risk of nephrogenic systemic fibrosis [36].
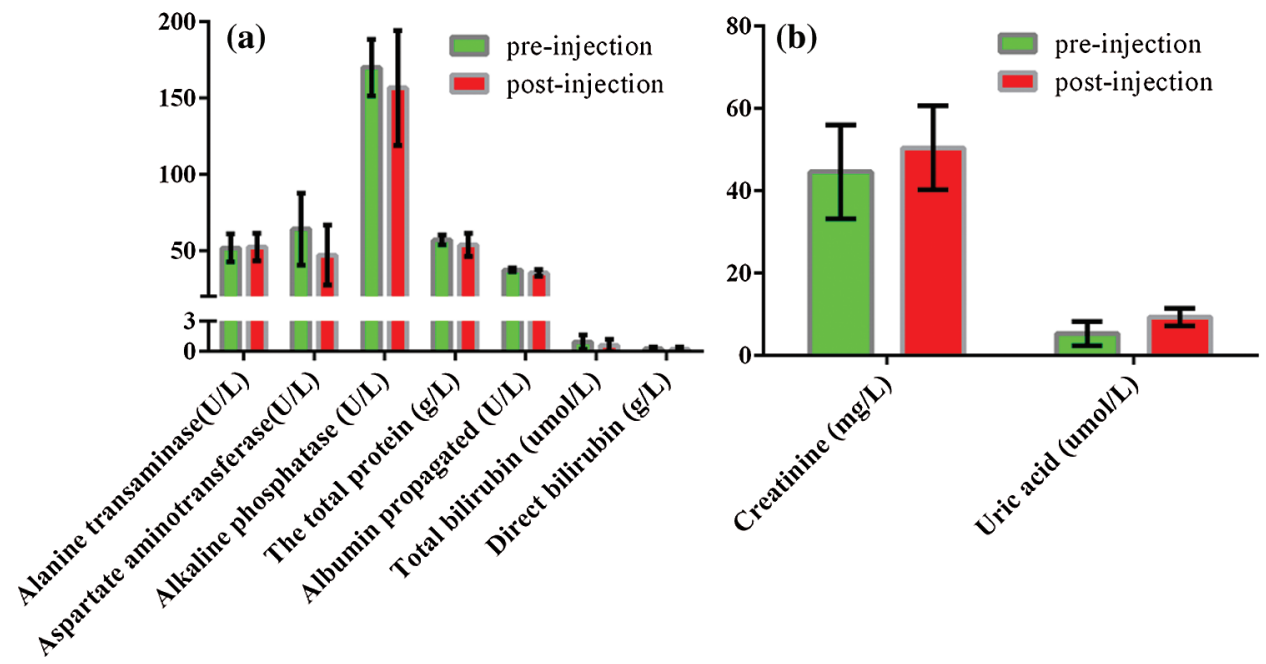

Figure 6: Biochemistry analyses before and $2 \mathrm{~h}$ after the administration of PFP/Lf-PLLA NBs. (a) liver function index, (b) renal function index. Data were reported as mean $\pm \mathrm{SD}(\mathrm{n}=5)$

\subsection{Ultrasonic Imaging in Vivo}

To evaluate the tumor enhanced ultrasound imaging capability of PFP/Lf-PLLA NBs in vivo, subcutaneous tumor animal models were constructed. As shown in Fig. 7a, before the administration of PFP/Lf-PLLA NBs, the area of the tumor was hypoechoic and dark region, and exhibited a blurred boundary with the surrounding tissue. However, when the PFP/Lf-PLLA NBs suspension was injected, there were obvious bright spots throughout the subcutaneous tumors, the gray-scale intensity increased and marked ultrasound imaging enhancement occurred immediately, the results were shown in Fig. $7 \mathrm{~b}$. The enhancement obtained using nanobubbles lasted for approximately $1.5 \mathrm{~h}$, indicating that the PFP/LfPLLA NBs were stable and long-lasting contrast ability enough for clinical ultrasonography.

PFP/Lf-PLLA NBs were prepared by the double emulsion method. PFP was a liquid state at room temperature, which was used as the $\mathrm{O}_{1}$ phase and encapsulated by an outer wall composed of the biodegradable polymer. As a result, the PFP stabilized by the outer layer could not diffuse away from the $\mathrm{NBs}$, furthermore, the F atom in PFP could form a hydrogen bond with the hydroxyl group in the chain segment of PLLA molecule [37,38], which caused the nanobubbles more stable and had long-lasting ultrasonic contrast ability. PFP is liquid at room temperature and atmospheric pressure. Under the action of thermal and mechanical factors, the wrapped PFP underwent phase transition from liquid to gas and triggered droplet to bubble transition. After the phase transition, the nanobubbles would change into larger nanobubbles or microbubbles. Gases of PFP encapsulated in the nanobubbles stabilized by elastic polymer walls are compressible. Under the action of ultrasound, the nanoscale bubbles grow and shrink in a rhythmic fashion, which will generate harmonic signals and contrast-enhanced imaging would be acquired $[39,40]$. 

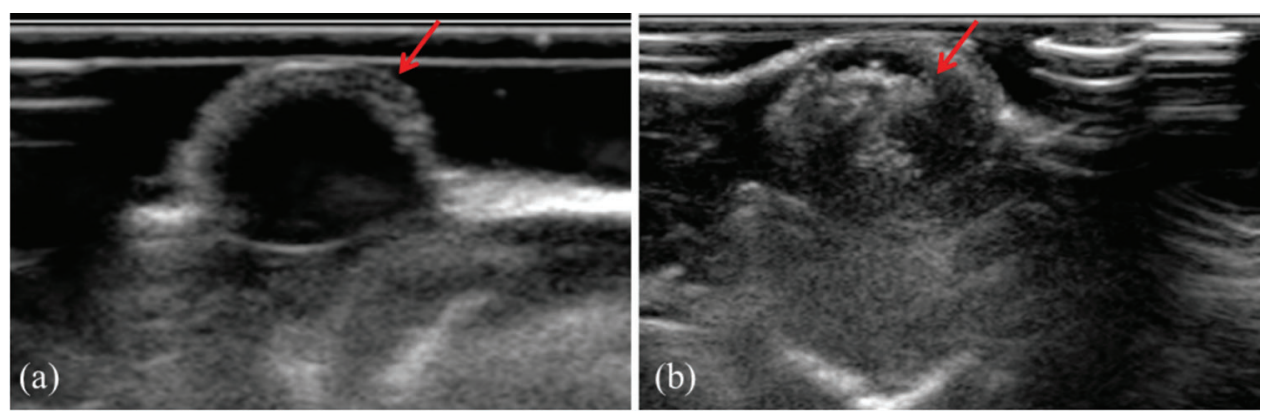

Figure 7: The effect of PFP/Lf-PLLA NBs on tumor imaging in vivo. (a) pre- and (b) post-injection of PFP/ Lf-PLLA NBs

\subsection{Magnetic Resonance Imaging in Vivo}

Fig. 8 was the T2-weighted magnetic resonance images of subcutaneous tumors before and after administration of PFP/Lf-PLLA NBs. Signal intensity was determined in several regions of interest (ROI) and measured before and after injection of the contrast agent. Before the administration of PFP/Lf-PLLA NBs, MR scans were performed using T2-weighted spin echo sequences, and obtained images were used as pre-injection, as shown in Fig. 8a, the tumor exhibited obscure boundaries to surrounding tissues. After injection of nanobubbles, it was clear that the signal intensity within the tumor was significantly decreased and become a dark signal area that was distinct from the surrounding tissue. A negative enhancement effect was observed in the tumor. The magnetic resonance signal intensity in the tumor region significantly decreased by $47 \%$ in comparison to the image of the pre-injection. The hypointense region with the tumor lesion was indicative of nanobubbles accumulation, which caused a reduction in signal intensity on T2-weighted images. The decreased signal lasted for hours.
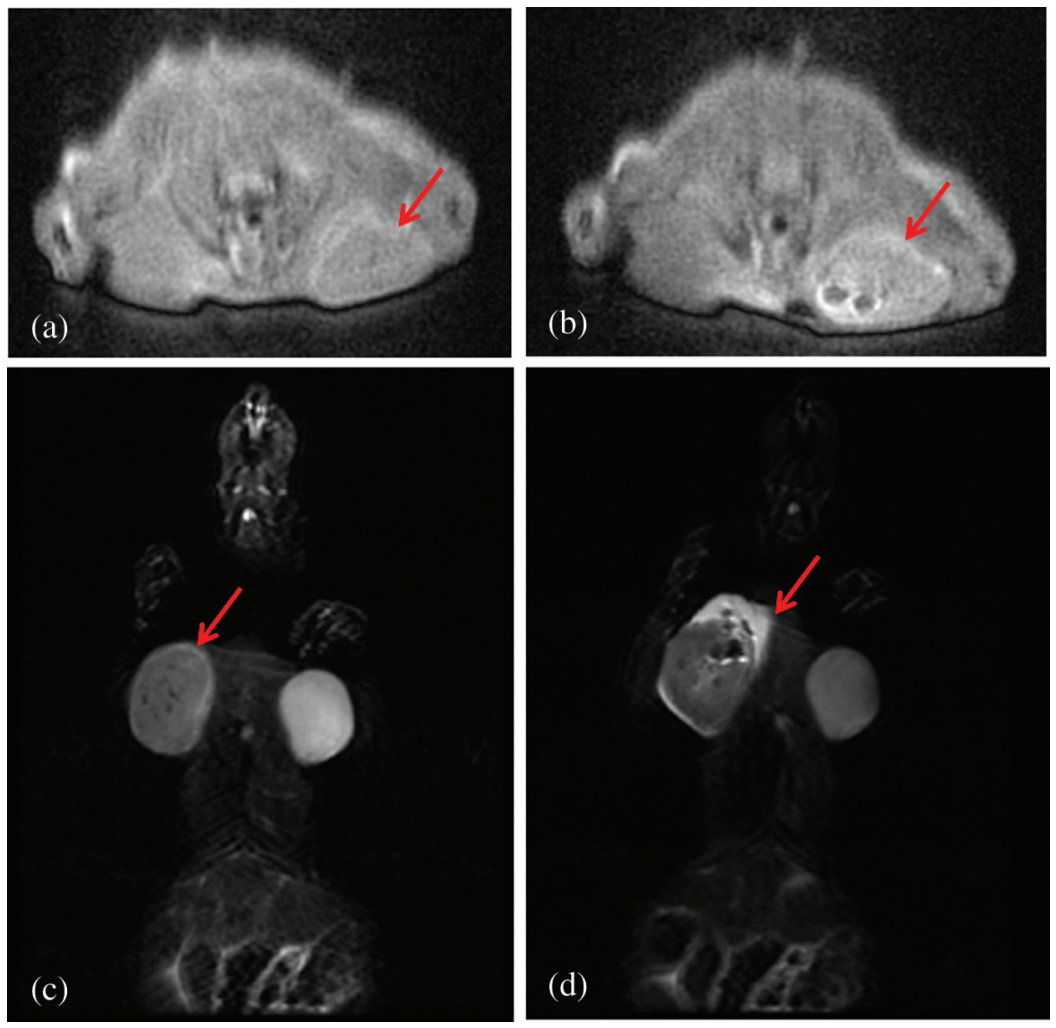

Figure 8: T2-weighted magnetic resonance images of the subcutaneous tumor pre- $(\mathrm{a}, \mathrm{c})$ and post-injection (b, d) of PFP/Lf-PLLA NBs. (a) and (b) are in the sagittal position,(c) and (d) are in the coronary position 
The signal intensity (SI) in the tumor on T2-weighted magnetic resonance images was measured with a RadiAnt DICOM Viewer software by defining the ratio of the mean signal intensity (the five points) in the region of interest (ROI) within the tumor area to the signal intensity of the surrounding muscle. The smaller the value of ratio was, the more serious the signal attenuation was, and the clearer the boundary with the surrounding area was, which showed that the tumor area was the dark signal area. The SI (tumor/muscle) of pre-injection were 190.2 $\pm 2.566 \%$, and the SI (tumor/muscle) of post-injection were $143.5 \pm 9.660 \%$ $(\mathrm{n}=5)$. The change of SI(tumor/muscle) was statistically significant before and after administration of PFP/Lf-PLLA NBs $(P<0.05)$. The results were shown in the following Fig. 9. These results indicated that PFP/Lf-PLLA NBs exhibited the ability to improve performance in magnetic resonance imaging and might be used in enhanced magnetic resonance imaging.

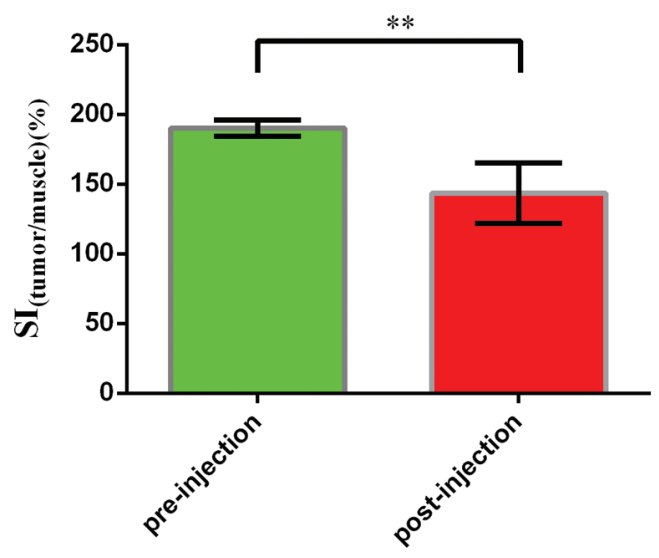

Figure 9: The signal intensity pre- and post-injection of PFP/Lf-PLLA NBs. Data are presented as the mean $\pm \mathrm{SD}, * * P<0.01$

\section{Conclusions}

The study reported here investigated a novel nanoscale bubble using as a contrast agent for ultrasound/ magnetic resonance imaging. The PFP/Lf-PLLA NBs were prepared with a double-emulsion (water/oil/ water) and solvent-evaporation method. Liquid PFP was encapsulated with polylactic acid (PLLA) and formed a core-shell structure. The obtained PFP/Lf-PLLA NBs had good stability in vitro.

The PFP/Lf-PLLA NBs solution with different concentrations showed little cytotoxicity and a low hemolysis rate. They also had no obvious effect on cell growth and no obvious potential hepatic and renal toxicity. The results of cytotoxicity, hemolysis, cell migration, and blood biochemical tests showed that PFP/LF-PLLA NB had good biocompatibility.

Under the action of ultrasound, the PFP/Lf-PLLA NBs could enhance the ultrasonic imaging of tumor tissue. On the magnetic resonance imaging of tumor-bearing mice, the signal intensity in the region of interest was significantly decreased, and a negative enhancement effect was observed in the tumor. The results of ultrasound and magnetic resonance imaging in vivo indicated that the PFP/Lf-PLLA NBs possessed the ability of enhanced contrast imaging, which had great potential to be a contrast agent for tumor dual-modality imaging in vivo.

Funding Statement: This work was financially supported by Initial Scientific Research Fund of Ph.D. in Hubei University of Science and Technology (BK202120). 
Conflicts of Interest: The authors declare that they have no conflicts of interest to report regarding the present study.

\section{References}

1. Junn, J. C., Soderlund, K. A., Glastonbury, C. M. (2021). Imaging of head and neck cancer with CT, MRI, and US. Seminars in Nuclear Medicine, 51(1), 3-12. DOI 10.1053/j.semnuclmed.2020.07.005.

2. Du, J., Li, X. Y., Hu, H., Xu, L., Yang, S. P. et al. (2018). Preparation and imaging investigation of dual-targeted $\mathrm{C}_{3} \mathrm{~F}_{8}$-filled PLGA nanobubbles as a novel ultrasound contrast agent for breast cancer. Scientific Reports, 8(1), 3887. DOI 10.1038/s41598-018-21502-x.

3. Zhu, L., Wang, L., Liu, Y., Xu, D., Fang, K. et al. (2018). CAIX Aptamer-functionalized targeted nanobubbles for ultrasound molecular imaging of various tumors. International Journal of Nanomedicine, 13, 6481-6495. DOI 10.2147/IJN.S176287.

4. Liu, L., Liu, L., Li, Y., Huang, X., Gu, D. et al. (2019). Ultrasmall superparamagnetic nanoparticles targeting Eselectin: Synthesis and effects in mice in vitro and in vivo. International Journal of Nanomedicine, 14, 4517-4528. DOI 10.2147/IJN.S199571.

5. Gao, Y., Hernandez, C., Yuan, H. X., Lilly, J., Kota, P. et al. (2017). Ultrasound molecular imaging of ovarian cancer with CA-125 targeted nanobubble contrast agents. Nanomedicine: Nanotechnology, Biology, and Medicine, 13(7), 2159-2168. DOI 10.1016/j.nano.2017.06.001.

6. Cai, W., Lv, W., Feng, Y., Yang, H., Zhang, Y. et al. (2018). The therapeutic effect in gliomas of nanobubbles carrying siRNA combined with ultrasound-targeted destruction. International Journal of Nanomedicine, 13, 6791-6807. DOI 10.2147/IJN.S164760.

7. Zhang, Y., Wan, C. F., Du, J., Dong, Q., Wang, Y. Y. et al. (2018). The in vitro study of her-2 targeted gold nanoshell liquid fluorocarbon poly lactic-co-glycolic acid ultrasound microcapsule for ultrasound imaging and breast tumor photothermal therapy. Journal of Biomaterials Science. Polymer Edition, 29(1), 57-73. DOI 10.1080/09205063.2017.1399003.

8. Cheung, J. S., Chow, A. M., Guo, H., Wu, E. X. (2009). Microbubbles as a novel contrast agent for brain MRI. NeuroImage, 46(3), 658-664. DOI 10.1016/j.neuroimage.2009.02.037.

9. Alexander, A. L., McCreey, T. T., Barrette, T. R., Gmitro, A. F., Unger, E. C. (1996). Microbubbles as novel pressure-sensitive MR contrast agents. Magnetic Resonance in Medicine, 356(6), 801-806. DOI 10.1002/ mrm.1910350603.

10. Dharmakumar, R., Plewes, D. B., Wright, G. A. (2002). On the parameters affecting the sensitivity of MR measures of pressure with microbubbles. Magnetic Resonance in Medicine, 47(2), 264-273. DOI 10.1002/ mrm. 10075 .

11. Dharmakumar, R., Plewes, D. B., Wright, G. A. (2005). A novel microbubble construct for intracardiac or intravascular MR manometry: A theoretical study. Physics in Medicine and Biology, 50(20), 4745-4762. DOI 10.1088/0031-9155/50/20/001.

12. Wong, K. K., Huang, I., Kim, Y. R., Tang, H., Yang, E. S. et al. (2004). In vivo study of microbubbles as an MR susceptibility contrast agent. Magnetic Resonance in Medicine: Official Journal of the Society of Magnetic Resonance in Medicine, 52(3), 445-452. DOI 10.1002/mrm.20181.

13. Yin, T., Wang, P., Zheng, R., Zheng, B., Cheng, D. et al. (2012). Nanobubbles for enhanced ultrasound imaging of tumors. International Journal of Nanomedicine, 7, 895-904. DOI 10.2147/IJN.S28830.

14. Zhou, T., Cai, W., Yang, H., Zhang, H., Hao, M. et al. (2018). Annexin V conjugated nanobubbles: A novel ultrasound contrast agent for in vivo assessment of the apoptotic response in cancer therapy. Journal of Controlled Release, 276, 113-124. DOI 10.1016/j.jconrel.2018.03.008.

15. Wu, M., Wang, Y., Wang, Y., Zhang, M., Luo, Y. et al. (2017). Paclitaxel-loaded and A10-3.2 aptamer-targeted poly(lactide-co-glycolic acid) nanobubbles for ultrasound imaging and therapy of prostate cancer. International Journal of Nanomedicine, 12, 5313-5330. DOI 10.2147/IJN.S136032. 
16. Song, Z., Wang, Z., Shen, J., Xu, S., Hu, Z. (2017). Nerve growth factor delivery by ultrasound-mediated nanobubble destruction as a treatment for acute spinal cord injury in rats. International Journal of Nanomedicine, 12, 1717-1729. DOI 10.2147/IJN.S128848.

17. Cai, W. B., Yang, H. L., Zhang, J., Yin, J. K., Yang, Y. L. et al. (2015). The optimized fabrication of nanobubbles as ultrasound contrast agents for tumor imaging. Scientific Reports, 5, 13725. DOI 10.1038/srep13725.

18. Liu, R., Tang, J., Xu, Y., Dai, Z. (2019). Bioluminescence imaging of inflammation in vivo based on bioluminescence and fluorescence resonance energy transfer using nanobubble ultrasound contrast agent. ACS Nano, 13(5), 5124-5132. DOI 10.1021/acsnano.8b08359.

19. Shin, J. E., Ogunyankin, M. O., Zasadzinski, J. A. (2019). Perfluoroheptane-loaded hollow gold nanoshells reduce nanobubble threshold flux. Small, 15(7), e1804476. DOI 10.1002/smll.201804476.

20. Wu, M., Zhao, H., Guo, L., Wang, Y., Song, J. et al. (2018). Ultrasound-mediated nanobubble destruction (UMND) facilitates the delivery of A10-3.2 aptamer targeted and siRNA-loaded cationic nanobubbles for therapy of prostate cancer. Drug Delivery, 25(1), 226-240. DOI 10.1080/10717544.2017.1422300.

21. Fan, X., Guo, Y., Wang, L., Xiong, X., Zhu, L. et al. (2016). Diagnosis of prostate cancer using anti-PSMA aptamer A10-3.2-oriented lipid nanobubbles. International Journal of Nanomedicine, 11, 3939-3950. DOI 10.2147/IJN. S112951.

22. Guvener, N., Appold, L., de Lorenzi, F., Golombek, S. K., Rizzo, L. Y. et al. (2017). Recent advances in ultrasound-based diagnosis and therapy with micro- and nanometer-sized formulations. Methods, 130, 4-13. DOI 10.1016/j.ymeth.2017.05.018.

23. Duan, S., Guo, L., Shi, D., Shang, M., Meng, D. et al. (2017). Development of a novel folate-modified nanobubbles with improved targeting ability to tumor cells. Ultrasonics Sonochemistry, 37, 235-243. DOI 10.1016/j.ultsonch.2017.01.013.

24. Yin, T., Wang, P., Li, J., Wang, Y., Zheng, B. et al. (2014). Tumor-penetrating codelivery of siRNA and paclitaxel with ultrasound-responsive nanobubbles hetero-assembled from polymeric micelles and liposomes. Biomaterials, 35(22), 5932-5943. DOI 10.1016/j.biomaterials.2014.03.072.

25. Li, J., Tian, Y., Shan, D., Gong, A., Zeng, L. et al. (2017). Neuropeptide Y Y1 receptor-mediated biodegradable photoluminescent nanobubbles as ultrasound contrast agents for targeted breast cancer imaging. Biomaterials, 116, 106-117. DOI 10.1016/j.biomaterials.2016.11.028.

26. Lou, S. T., Ouyang, Z. Q., Zhang, Y., Li, X. J., Hu, J. et al. (2000). Nanobubbles on solid surface imaged by atomic force microscopy. Journal of Vacuum Science \& Technology B: Microelectronics and Nanometer Structures, 18(5), 2573. DOI 10.1116/1.1289925.

27. Vannozzi, L., Gouveia, P., Pingue, P., Canale, C., Ricotti, L. (2021). Novel ultrathin films based on a blend of PEGb-PCL and PLLA and doped with ZnO nanoparticles. ACS Applied Materials \& Interfaces, 12(19), 21398-21410. DOI 10.1021/acsami.0c00154.

28. Nanaki, S. G., Spyrou, K., Bekiari, C., Veneti, P., Baroud, T. N. et al. (2020). Hierarchical porous Carbon-PLLA and PLGA hybrid nanoparticles for intranasal delivery of galantamine for Alzheimer's disease therapy. Pharmaceutics, 12(3). DOI 10.3390/pharmaceutics12030227.

29. Pappu, A., Pickering, K. L., Thakur, V. K. (2019). Manufacturing and characterization of sustainable hybrid composites using sisal and hemp fibres as reinforcement of poly (lactic acid) via injection moulding. Industrial Crops and Products, 137, 260-269. DOI 10.1016/j.indcrop.2019.05.040.

30. Leluk, K., Frackowiak, S., Ludwiczak, J., Rydzkowski, T., Thakur, V. K. (2020). The impact of filler geometry on polylactic acid-based sustainable polymer composites. Molecules, 26(1), 149. DOI 10.3390/molecules26010149.

31. Wang, M., Zhan, J., Xu, L., Wang, Y., Lu, D. et al. (2020). Synthesis and characterization of PLGA-PEG-pLGA based thermosensitive polyurethane micelles for potential drug delivery. Journal of Biomaterials Science. Polymer Edition, 32(5), 613-634. DOI 10.1080/09205063.2020.1854413.

32. Luo, B., Zhang, H., Liu, X., Rao, R., Wu, Y. et al. (2015). Novel DiR and SPIO nanoparticles embedded PEGpLGA nanobubbles as a multimodalimaging contrast agent. Bio-Medical Materials and Engineering, 26(Suppl 1), S911-S916. DOI 10.3233/BME-151384. 
33. Chu, X., Tang, J., Geng, Z., Wang, X., Huo, G. et al. (2019). Mechanical properties of sub-microbubbles with a nanoparticle-decorated polymer shell. Langmuir, 35(52), 17090-17095. DOI 10.1021/acs.langmuir.9b02930.

34. Ates, B., Koytepe, S., Ulu, A., Gurses, C., Thakur, V. K. (2020). Chemistry, structures, and advanced applications of nanocomposites from biorenewable resources. Chemical Reviews, 120(17), 9304-9362. DOI 10.1021/acs. chemrev.9b00553.

35. Wang, C. W., Yang, S. P., Hu, H., Du, J., Li, F. H. (2015). Synthesis, characterization and in vitro and in vivo investigation of $\mathrm{C}_{(3)} \mathrm{F}_{(8)}$-filled poly(lactic-co-glycolic acid) nanoparticles as an ultrasound contrast agent. Molecular Medicine Reports, 11(3), 1885-1890. DOI 10.3892/mmr.2014.2938.

36. Luo, B., Wang, S., Rao, R., Liu, X., Xu, H. et al. (2016). Conjugation magnetic PAEEP-pLLA nanoparticles with lactoferrin as a specific targeting MRI contrast agent for detection of brain glioma in rats. Nanoscale Research Letters, 11(1), 227-238. DOI 10.1186/s11671-016-1421-x.

37. Dian, L. H., Hu, Y. J., Lin, J. Y., Zhang, J. Y., Yan, Y. et al. (2018). Fabrication of paclitaxel hybrid nanomicelles to treat resistant breast cancer via oral administration. International Journal of Nanomedicine, 13, 719-731. DOI 10.2147/IJN.S150140.

38. Yen, C. C., Chang, C. W., Hsu, M. C., Wu, Y. T. (2017). Self-nanoemulsifying drug delivery system for resveratrol: Enhanced oral bioavailability and reduced physical fatigue in rats. International Journal of Molecular Sciences, 18(9), 1853. DOI 10.3390/ijms18091853.

39. Wang, H., Ding, L., Xu, F., He, L., Ye, L. et al. (2021). Construction of novel amphiphilic chitosan-polylactide graft copolymer nanodroplets for contrast enhanced ultrasound tumor imaging. Journal of Biomaterials Applications, 8853282211011766. DOI 10.1177/08853282211011766.

40. Xiao, R., Zhao, Z., Chen, J., He, L., Wang, H. et al. (2020). Preparation and ultrasonic imaging investigation of perfluoropentane-filled polylactic acid nanobubbles as a novel targeted ultrasound contrast agent. Frontiers in Materials, 7, 549002. DOI 10.3389/fmats.2020.549002. 